\title{
Lubrizol's experiences with DAM
}

\author{
Robert Watts \\ joined Lubrizol in 1996. In 2001 he helped to set up the digital asset management (DAM) system at Lubrizol's headquarters in \\ Wickliffe, Ohio and their European research and development site at Hazelwood, UK. In January 2004 he was given overall \\ responsibility for the global asset library. Prior to joining Lubrizol, Robert was employed at a photographic laboratory where he \\ developed a computer control system for the laboratory's photographic printers. He has 25 years' photographic and computer systems \\ experience. Robert is a member of the Association for Information and Image Management (AllM) and he participated in the Taxonomy \\ and Metadata Strategies question and answer panel at this year's DAM symposium in London.
}

Keywords: taxonomy, IT, images, metadata, keyword, FotoWeb

Abstract This paper documents the evolution of one company's asset management system from a filing cabinet, into a 500Gb capacity, multiple site, global system, allowing instant access for their employees to any of the assets from anywhere in the world.

The Lubrizol Corporation is a global provider of specialty chemicals and materials for a wide variety of markets and end-use applications, such as transportation-related fluids and industrial lubricants. Lubrizol also makes ingredients and additives for personal care products and pharmaceuticals; polymer additives and plastics technology; specialty resins and additives for performance coatings; and additives for the food and beverage industry. Headquartered in Wickliffe, Ohio, Lubrizol operates manufacturing facilities in 22 countries, as well as sales and technical offices around the world, with more than 7,800 employees.

Lubrizol has three research centers, in Wickliffe, Ohio, USA, Hazelwood, UK and Kinuura, Japan. Most of our engine testing is done in these research centers. These are either on-site static bench tests or field tests of vehicles and machinery (almost anything involving an internal combustion engine). After the test is run, the engine is stripped down and the various components, pistons, valves, etc, are photographed. It's at this point I get involved in the process.

When I joined Lubrizol in 1996, all of Lubrizol's photography was still on negative with hundreds of thousands of negatives stored in filing cabinets. The paper-based filing system utilized the individual test numbers and Lubrizol market segment identifications to aid searching.

We had over 50,000 promotional images and 200,000 test images on negative going back over 40 years. In those days we didn't call it an asset management system, we called it a filing cabinet.
The way that users searched for images was quite different. Searching for a test image was straightforward. An engineer would request a photograph of a particular test that had been run using a particular oil sample. Every test that we run has a unique number and the negatives were filed according to that number. So it was relatively simple to find the negatives and print off the required photographs. The person requesting the pictures would usually get them back within a day.

The way that users would search for promotion images was completely different. In this case the user isn't looking for a particular photograph but rather an image that will best illustrate whatever they are trying to say. The only catalogue for the promotional images was a notebook with the relevant Lubrizol segment area and a short description and date for each image. Consequently if the design team needed an image of a Volvo truck on a bridge they would have to look through thousands of contact sheets for a suitable image.

As a result we tended to use the same few images over and over again. In addition the design team would usually scan the negative at a suitable resolution for each particular job. If that image was needed in a large size then the negative would have to be scanned again. This was costing both time and money and because of all the effort and trouble involved in looking through thousands of contact sheets, it meant that many of our images were simply never used. It was obvious that something needed to be done. 
In 1998 the communications team at Hazelwood purchased a copy of Portfolio 3. At the time our only thought was to produce a searchable catalog of images. We also adopted a strategy of always scanning the negatives at the maximum resolution possible to avoid repeat scanning. Although we originally envisioned only using Portfolio within the department, it was soon realized that we could offer a service to the rest of the site by placing the promotional catalogue on a server and giving everybody on the site a copy of the Portfolio Browser. Everyone was given access to low resolution images so that they could put together their own PowerPoint presentations. There were two main advantages in doing this. First it meant that our designers weren't spending lots of valuable time in searching contact sheets for images. Secondly, our customers could search our library themselves and find images within minutes instead of sending in a request and then waiting a day or two for a response (which might not have been the image they were after). This proved to be so successful that we started to think that maybe this was a service that we could offer globally.

It was at about this time that Wickliffe decided to switch from negative to digital photography. However, before this could be done, a storage and retrieval system needed to be put in place. The driving force behind this decision was the test photography, which outnumbers the promotional photography by about ten to one, however, the promotional photography would also be able to take advantage of the new system.

Because of the large number of files involved, the Wickliffe team opted for a dedicated file server and Chuckwalla software. After a few teething problems they had Chuckwalla up and running by mid-1999. In 2000 it was decided that Lubrizol needed a global solution for digital asset management (DAM), so a joint task group from both Wickliffe and Hazelwood was put together to look into combining the two DAM systems.

It was then that we noticed that the two systems were totally incompatible. Not only were the metadata stored differently - Portfolio embedded the data within the file, while Chuckwalla stored the data in a separate database, but even the nature of the metadata was different. The data that we recorded from our engine tests in Hazelwood were not always the same as the data that Wickliffe recorded from their tests, as the USA and EU have different regularity requirements for emissions etc; in addition, the record retention requirements differ between the USA and the EU. ${ }^{1}$

We reviewed the existing systems to compare advantages and disadvantages. After evaluating numerous systems it was decided to opt for the FotoWare suite of programs which combined user-friendliness with high-volume capacity. It also came with a web front-end called "FotoWeb".

This meant that both sites had to scrap their existing systems and start over again, but it was felt that it was worth it to get a truly global DAM system in place. We also looked at the metadata that both sites collected and initiated a project to harmonize the data. After about six months the team had produced a document called "Keyword Conventions" (we didn't call it a taxonomy then). Among some of the things that we had to consider was the fact that although the metadata from mechanical test laboratories in Wickliffe and Hazelwood sometimes differed we still needed to be able to access the Wickliffe images from Hazelwood and vice versa; in addition, we had to consider the difference in both UK and US spelling (eg colour/color, harbour/harbor) and terms (eg boot/trunk, bonnet/hood). ${ }^{2}$

In September 2001 the asset library went online in Wickliffe and followed in Hazelwood a few months later. Initially there were a few teething problems, not the least of which was FotoWeb's unfortunate tendency to return a different preview to the thumbnail clicked on, as well as some IT issues involved in linking the two servers in the US and UK into a combined system. $^{3}$

As before, the way that users search the test and promotional libraries differs. The only metadata that the test images need are, test number, oil sample number, test type, test laboratory, test date, test hours and test miles. Engineers can now access the test library from their PCs from anywhere in the world. They can search for the test number, download the images for use in a PowerPoint presentation, or print out hard copies as required. Instead of having to wait a day or so (longer if they are 
off-site) they can now get their images within seconds.

The promotional library is a more interesting situation, because you never know quite what the users are looking for. In fact, sometimes the users themselves don't know quite what they are looking for.

Once the system was up and running we found the system logs were very useful for refining our taxonomy, as well as determining which kind of images we needed to photograph. For instance, if users were searching for strimmer and not getting any hits it could be because we'd used the term weed eater in the metadata, or it could simply be that there were no pictures of weed eaters or strimmers in the library. Either way it is useful information to have, in the first instance we could amend the taxonomy, in the second instance, if the demand is high enough, we could go out and photograph some strimmers.

The way that synonyms are handled is through a thesaurus which contains a list of the preferred terms (terms that are recommended for a certain concept) and the accepted terms (terms that are not strictly recommended but are used and accepted). If the user was to search for lorry (accepted term) the thesaurus would translate that into truck (preferred term). The actual image is only tagged with the preferred term, in this case, truck. We also have to consider how the end user would classify images themselves. For instance, in our library we have an image of $L e$ Pont de Normandie. Strictly speaking, this is a cable-stayed bridge, however, the layman might well think of it as a suspension bridge, so suspension bridge is included in the keywords as well as cable-stayed bridge, although this is, strictly speaking, incorrect. Another example could be St. Stephen's Tower, most people would be more familiar with the name Big Ben, although this term refers neither to the tower or the clock but to the hour bell.

The taxonomy that we have developed is divided up into five facets (a facet is a subtaxonomy within the overall taxonomy see Figure 1).

The first facet refers to the Lubrizol market segment that relates to the image. PC is passenger car motor oil, HD is heavy duty diesel

\section{Lubrizol Taxonomy}

Facet Categories
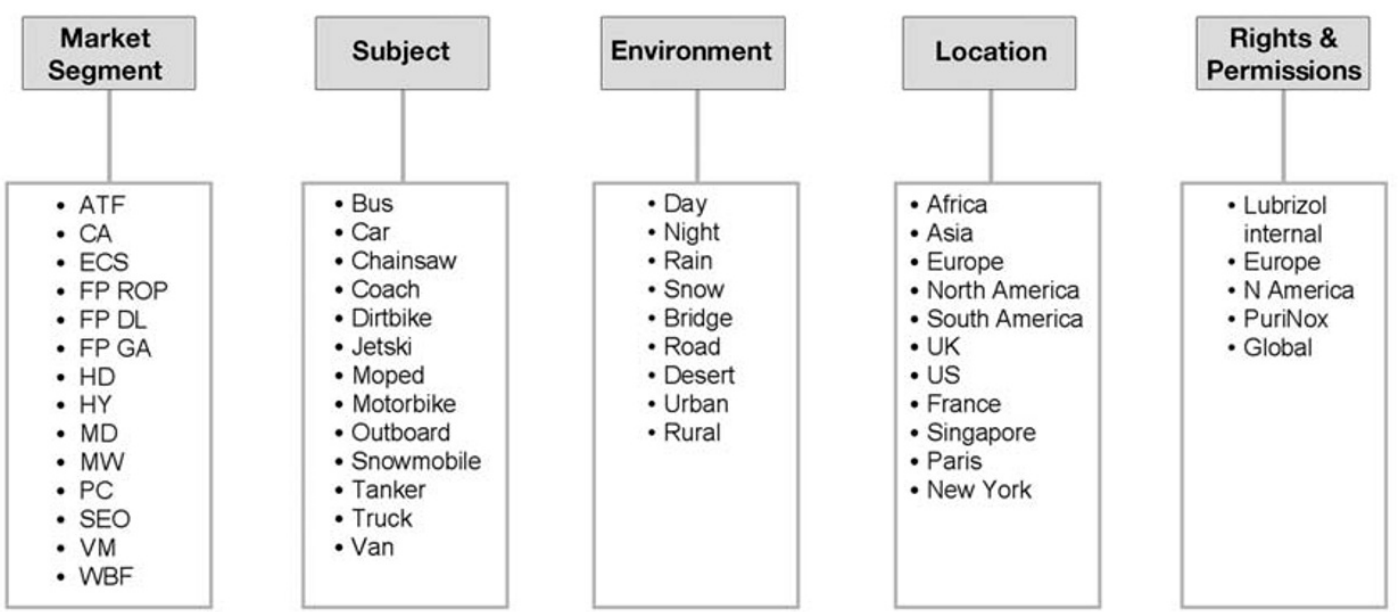

Figure 1: Example of Lubrizol's taxonomy 
oil (terms used by Lubrizol). It is possible that an image can belong to more than one market segment. For example, a picture of traffic on a motorway containing both cars and trucks could equally apply to PC and HD. This simply wasn't possible with the manual filing system where the negatives could only be filed under one market segment. This is known as allowing a child to have more than one parent.

The second facet is the actual subject of the image. The vocabulary here contains only the preferred terms (of course, the example above does not contain our entire controlled vocabulary, which runs to many hundreds of keywords). If the subject of an image is not within the controlled vocabulary (eg an elephant) then the person keying in the metadata is free to add any keywords that they feel are appropriate.

The third facet is the environment in which the subject was photographed. We are a little more relaxed here as to the vocabulary used (it's not possible to have a controlled vocabulary of all possible environments) and the person tagging the image is encouraged to be as descriptive as possible.

The fourth facet is simply the continent, country, city/town where the picture was taken as well as the date that the picture was taken.

The final facet is the circumstances under which we can use the image. As we have our own photographers and take most of our pictures, this is not usually a problem. Occasionally, however, we buy in our images, or we will have photographed something that is to be used in a particular campaign and only that campaign.

All keywords within the controlled vocabulary are added to the image via a dropdown list, so the person tagging the image doesn't have to do too much typing (this also helps cut down on typos). Any keywords that are not within the controlled vocabulary are typed in manually.

The taxonomy is not set in stone, as the business changes and as user requirements change the taxonomy has to change to meet the new conditions. Major changes to an existing taxonomy can be quite expensive and timeconsuming to implement, however, so we prefer to adapt and tweak where possible. ${ }^{4}$

The current system stores approximately
25,000 test images from Hazelwood, 50,000 test images from Wickliffe, 7,000 promotional images, 14,000 PowerPoint presentations, 1,200 portraits and 20,000 PDFs of our test reports. In total 128,000 files and any one of our 7,000 employees can find and retrieve any one of those files within seconds, from any of our facilities throughout the world.

Has it all been worth it? Yes, most definitely! True there were times when we were tearing our hair out and we thought that it would never work, but now that it does the advantages are enormous. Engineers and chemists can now get instant access to our images instead of waiting days. The design team can search through hundreds of images for that one special image, from their desktop instead of having to get themselves over to our negative library. We are also able to repurpose images that we already own, instead of having to go out and either purchase an image or photograph it ourselves. For example, we used the existing database images for our latest annual report, saving Lubrizol thousands of dollars, without any loss in quality, meeting our usual high standards.

As to future expansion, we are looking for ways we can broaden access to the asset library via the world wide web and thereby give our customers, suppliers and business partners access to our images. We are also looking at the storage of video clips. Because of the file sizes involved and bandwidth issues, online storage of an entire video is not practical with today's technology, but in the future who knows?

\section{LESSONS LEARNED}

1. Before embarking on a DAM project find out what the rest of the organization is doing.

2. You cannot impose a taxonomy from the top down, it's no good insisting that it's a scooter if your users are searching for a moped.

3. Involve IT as early as possible, for some strange reason they don't like holes in their firewalls.

4. As far as possible try to get the taxonomy right before tagging thousands of images. Try it out on a couple of hundred images first, with a small user test group. 